\title{
Correction to: Performance Improvements on SNS and HFIR Instrument Data Reduction Workflows Using Mantid
}

\author{
William F. Godoy, Peter F. Peterson, Steven E. Hahn, John Hetrick,
}

Mathieu Doucet, and Jay J. Billings

\section{Correction to: \\ Chapter "Performance Improvements on SNS and HFIR Instrument Data Reduction Workflows Using Mantid" in: J. Nichols et al. (Eds.): Driving Scientific and Engineering Discoveries Through the Convergence of HPC, Big Data and AI, CCIS 1315, https://doi.org/10.1007/978-3-030-63393-6_12}

In the originally published version of the chapter 12 , the reference 21 contained a mistake in the name of the author. The author's name in the reference was changed to S. Hahn. 